\title{
Thermodynamic Behavior of Friedmann Equation at Apparent Horizon of FRW Universe
}

\author{
M. Akbar* and Rong-Gen Cai ${ }^{\dagger}$ \\ Institute of Theoretical Physics, Chinese Academy of Sciences, \\ P.O. Box 2735, Beijing 100080, China
}

\begin{abstract}
It is shown that the differential form of Friedmann equation of a FRW universe can be rewritten as the first law of thermodynamics $d E=T d S+W d V$ at apparent horizon, where $E=\rho V$ is the total energy of matter inside the apparent horizon, $V$ is the volume inside the apparent horizon, $W=(\rho-P) / 2$ is the work density, $\rho$ and $P$ are energy density and pressure of matter in the universe, respectively. From the thermodynamic identity one can derive that the apparent horizon $\tilde{r}_{A}$ has associated entropy $S=A / 4 G$ and temperature $T=\kappa / 2 \pi$ in Einstein general relativity, where $A$ is the area of apparent horizon and $\kappa$ is the surface gravity at apparent horizon of FRW universe. We extend our procedure to the Gauss-Bonnet gravity and more general Lovelock gravity and show that the differential form of Friedmann equations in these gravities can also be written as $d E=T d S+W d V$ at the apparent horizon of FRW universe with entropy $S$ being given by expression previously known via black hole thermodynamics.
\end{abstract}

PACS numbers:

\footnotetext{
* Email address: akbar@itp.ac.cn

† Email address: cairg@itp.ac.cn
} 


\section{INTRODUCTION}

Semiclassical quantum properties of black hole can be analyzed in the context of quantum field theory in curved backgrounds, where matter is described by quantum field theory while gravity enters as a classical background. In this framework, it was discovered that black holes can emit Hawking radiation with a temperature proportional to its surface gravity at the black hole horizon and black hole has an entropy proportional to its horizon area [1, 2]. The Hawking temperature and horizon entropy together with the black hole mass obey the first law of black hole thermodynamics 3]. The gravitational entropy of black hole in Einstein gravity is given by

$$
S=\frac{A}{4 G}
$$

where $A$ is the black hole horizon area and the units are such that $c=\hbar=k=1$. The Hawking temperature is given by

$$
T_{H}=\frac{\kappa}{2 \pi}
$$

where $\kappa$ is the surface gravity of the black hole. The Hawking temperature together with black hole entropy is related by the first law of black hole thermodynamics $T d S=d M$, where $M$ is the black hole mass (For a more general Kerr-Newman black hole, the first law is $d M=T d S+\Omega d J+$ $\Phi d Q)$. The black hole thermodynamics and the statistical property of black hole entropy have been investigated from many different points of view in literature [4]. Nowadays it is widely believed that a black hole behaves like an ordinary thermodynamic system and satisfies laws of thermodynamics. If one identities the black hole mass $M$ as the energy $E$, obviously, a work term is absent in the first law of black hole thermodynamics $d E=T d S$. To remedy this drawback, More recently, Paranjape Sarkar and Padmanabhan [5] have considered a special kind of spherically symmetric black hole spacetimes, and found that it is possible to interpret Einstein's equations as the thermodynamic identity $T d S=d E+P d V$ by considering black hole horizon as the system boundary. For related discussions on this issue see, for example, references in [6].

On the other hand, the thermodynamical properties of the black hole horizon can be generalized to the space-time horizons other than black hole horizon. For example, the de Sitter space-time with radius $\ell$, there is a cosmological event horizon. This horizon, like black hole horizon, can be regarded as a thermodynamical system [7] associated with the Hawking temperature $T=1 / 2 \pi \ell$ and entropy $S=A / 4 G$, where $A=4 \pi \ell^{2}$ is the cosmological horizon area of the de Sitter spacetime. For an asymptotic de Sitter space, like Schwarzschild-de Sitter space-time, there still exists 
the cosmological horizon which behaves like a black hole horizon with entropy proportional to the area of the cosmological horizon and whose Hawking temperature is given by $T=\kappa / 2 \pi$, where $\kappa$ is the surface gravity of the cosmological horizon. It is easy to verify that the cosmological horizons of these space-times satisfy the first law of black hole thermodynamics of the form $T d S=-d M$ [8], where the minus appears due to the fact that when the black hole mass $M$ increases, the cosmological horizon entropy decreases.

Indeed black hole physics implies that there is some relation between the first law of thermodynamics and Einstein's equations. Jacobson [9] is the first one to seriously investigate such a relation. Jacobson finds that it is indeed possible to derive the Einstein's equations from the proportionality of entropy to the horizon area together with the fundamental relation $\delta Q=T d S$, assuming the relation holds for all local Rindler causal horizons through each spacetime point. Here $\delta Q$ and $T$ are the energy flux and Unruh temperature seen by an accelerated observer just inside the horizon.

In Ref. [10], one of the present authors and Kim are able to derive the Friedmann equations of an (n+1)-dimensional Friedman-Robertson-Walker (FRW) universe with any spatial curvature by applying the first law of thermodynamics $(T d S=-d E)$ to the apparent horizon of the FRW universe and by working out the heat flow through the apparent horizon. In the process, an ansatz is made: suppose that the apparent horizon has temperature and entropy expressed by

$$
T=\frac{1}{2 \pi \tilde{r}_{A}}, \quad S=\frac{A}{4 G}
$$

where $A$ is the area of the apparent horizon. Also by using the entropy expression of a static spherically symmetric black hole in the Gauss-Bonnet gravity and in more general Lovelock gravity, they reproduce the corresponding Friedmann equations in each gravity. The possible extensions to the scalar-tensor gravity and $f(R)$ gravity theory have been studied in reference [11]. In the cosmological setting, related discussions see also, [12, 13, 14, 15].

Note that in the process of deriving the Friedmann equations, $-d E$ is interpreted as the amount of energy flux crossing the apparent horizon within an infinitesimal time interval $d t$ and the horizon radius is assumed to be not changed during the internal. On the other hand, we know that the Friedmann equations of FRW universe are the field equations with a source of perfect fluid. In this cosmological setup, different from the case of black hole spacetimes discussed in [5], there is a welldefined concept of pressure $P$ and energy density $\rho$. Therefore, it is very interesting to see whether it is possible to rewrite the Friedmann equations as a thermodynamical identity $T d S=d E+P d V$ at apparent horizon of the FRW universe? To resolve this issue we develop a procedure to study the thermodynamical properties of Friedmann equations at apparent horizon of a FRW universe 
and show that by employing Misner-Sharp energy relation inside a sphere of radius $\tilde{r}_{A}$ of apparent horizon, it is indeed possible to rewrite the differential form of the Friedman equations as the form of the first law of thermodynamic $d E=T d S+W d V$ with $W=(\rho-P) / 2$, where $\rho$ and $P$ are energy density and pressure of matter in the universe. In the case of Einstein gravity, we find that the horizon entropy $S$ is proportional to the apparent horizon area and the temperature $T$ is given by $T=\kappa / 2 \pi$ with surface gravity at the apparent horizon, and the Misner-Sharp energy is just the total energy of matter inside the apparent horizon $(E=\rho V)$. We extend this procedure to the Gauss-Bonnet gravity and in more general Lovelock gravity, and verify that the Friedmann equations at apparent horizon can also be rewritten as a universal form $d E=T d S+W d V$ in these gravities. It is important to mention here that one can pick up the expressions for entropy $S$ from the identity $d E=T d S+W d V$ obtained from the field equations at apparent horizon, which agrees with the expression previously derived by black hole thermodynamics. In addition, for the Gauss-Bonnet gravity and Lovelock gravity, the energy in the first law is not the Misner-Sharp energy, but the total energy $(\rho V)$ of matter inside the apparent horizon. This will be seen shortly.

This paper is organized as follows. In Sec. II, we shall summarize the procedure constructed to study the thermodynamic properties of the apparent horizon through the Friedmann equations of a FRW universe. In Sec. III, we shall apply our procedure to the field equations of FRW universe in Einstein gravity. We shall extend our procedure to the Gauss-Bonnet gravity and to more general Lovelock gravity in Sec. IV and Sec. V, respectively. Finally in Sec. VI, we shall conclude our results with some discussions.

\section{THE PROCEDURE}

We consider a spatially homogenous and isotropic universe described by the FRW metric. The line element of an $(n+1)$-dimensional FRW universe is represented by

$$
d s^{2}=-d t^{2}+a^{2}(t) \gamma_{i j} d x^{i} d x^{j}
$$

where the $n$-dimensional spatial hypersurfaces with negative, zero or positive curvature are parameterized by $k=-1,0$ and 1 , respectively, and $a(t)$ is the scale factor of the universe with $t$ being the cosmic time. The metric $\gamma_{i j}$ is given by

$$
\gamma_{i j} d x^{i} d x^{j}=\frac{d r^{2}}{1-k r^{2}}+r^{2} d \Omega_{n-1}^{2}
$$

Here $d \Omega_{n-1}^{2}$ is the metric of $(n-1)$-dimensional sphere with unit radius and the spatial curvature constant $k=1,0$ and -1 correspond to a closed, flat and open universe, respectively. Using 
spherical symmetry, the metric (44) can be re-written as

$$
d s^{2}=h_{a b} d x^{a} d x^{b}+\tilde{r}^{2} d \Omega_{n-1}^{2},
$$

where $\tilde{r}=a(t) r$ and $x^{0}=t, x^{1}=r$ and the two dimensional metric $h_{a b}=\operatorname{diag}\left(-1, a^{2} / 1-k r^{2}\right)$. The dynamical apparent horizon is determined by the relation $h^{a b} \partial_{a} \tilde{r} \partial_{b} \tilde{r}=0$, which implies that the vector $\nabla \tilde{r}$ is null on the apparent horizon surface. The explicit evaluation of the apparent horizon for the FRW universe gives the apparent horizon radius

$$
\tilde{r}_{A}=1 / \sqrt{H^{2}+k / a^{2}}
$$

The associated temperature $T=\kappa / 2 \pi$ at the apparent horizon is determined through the surface gravity

$$
\kappa=\frac{1}{2 \sqrt{-h}} \partial_{a}\left(\sqrt{-h} h^{a b} \partial_{b} \tilde{r}\right)
$$

The explicit evolution of the surface gravity at apparent horizon of FRW universe reads [10]

$$
\kappa=-\frac{1}{\tilde{r}_{A}}\left(1-\frac{\dot{\tilde{r}}_{A}}{2 H \tilde{r}_{A}}\right),
$$

where an over-dot denotes the derivative with respect to the cosmic time $t$. We now introduce the total energy $E$ inside a sphere of radius $\tilde{r}$ defined by

$$
E=\frac{n(n-1) \Omega_{n} \tilde{r}^{n-2}}{16 \pi G}\left(1-h^{a b} \partial_{a} \tilde{r} \partial_{b} \tilde{r}\right)
$$

where $V=\Omega_{n} \tilde{r}^{n}$ is the volume of an $n$-dimensional sphere with radius $\tilde{r}$ and $\Omega_{n}=\frac{\pi^{n / 2}}{\Gamma(n / 2+1)}$ being the volume of an $n$-dimensional unit ball. The total energy (10) is actually the direct $(n+1)$ dimensional generalization of the (3+1)-dimensional one, given by Misner and Sharp [16]. At the apparent horizon $\tilde{r}=\tilde{r}_{A}$, the term $h^{a b} \partial_{a} \tilde{r} \partial_{b} \tilde{r}=0$ in equation (10), therefore the total energy inside a sphere of radius $\tilde{r}_{A}$ is given by

$$
E=\frac{n(n-1) \Omega_{n}}{16 \pi G} \tilde{r}_{A}^{n-2}
$$

which agrees with the expression for the mass in the $(n+1)$-dimensional Schwarzschild black hole once the apparent horizon is replaced by the event horizon of the black hole. We consider the FRW universe as a thermodynamical system with apparent horizon surface as a boundary of the system. In general the radius of the apparent horizon $\tilde{r}_{A}$ is not constant but changes with time. Let $d \tilde{r}_{A}$ be an infinitesimal change in radius of the apparent horizon of FRW universe during a time of interval $d t$. This small displacement $d \tilde{r}_{A}$ in the radius of apparent horizon will cause a small change $d V$ in 
the volume $V$ of the apparent horizon. This leads to build up two spherical systems of space-time with radii $\tilde{r}_{A}$ and $\tilde{r}_{A}+d \tilde{r}_{A}$ having a common source $T_{\mu \nu}$ of perfect fluid with non-zero pressure $P$ and energy density $\rho$ near apparent horizon. Each space-time describing a thermodynamical system and satisfying Einstein equations, differs infinitesimally in the extensive variables volume, energy and entropy by $d V, d E$ and $d S$, respectively, while having same values the intensive variables temperature $T$ and pressure $P$. Thus, for these two space-times describing thermodynamical states, there must exist a certain relation relating these thermodynamic quantities. It turns out it is indeed the case. Mathematically, the main points of the procedure can be summarized as follows.

i) Write down the Friedmann equation of FRW universe in term of radius $\tilde{r}_{A}$ of the apparent horizon. Then taking its differential, one gets a new form of Friedmann equation called the differential form of the Friedmann equation. The differential form of the field equations describes how the changes near apparent horizon are related through the field equations.

ii) Multiply by a factor $n \Omega_{n} \tilde{r}_{A}\left(1-\frac{\dot{\tilde{r}}_{A}}{2 H \tilde{r}_{A}}\right)$ on both sides of the differential form of the Friedmann equation and then simplify it to get an equation of the form

$$
T d S=n \Omega_{n} \tilde{r}_{A}^{n}(\rho+P) H\left(1-\frac{\dot{\tilde{r}}_{A}}{2 H \tilde{r}_{A}}\right) d t,
$$

where $A=n \Omega_{n} \tilde{r}_{A}^{n-1}$ is the area of apparent horizon, $H$ denotes the Hubble parameter, $T=\kappa / 2 \pi$, and $\rho$ and $P$ are energy density and pressure of perfect fluid in the FRW universe, respectively.

iii) Write down the expression for the total energy $E$ for $(n+1)$-dimensional FRW universe inside a sphere of radius $\tilde{r}_{A}$ and then find out $d E$, an infinitesimal change in energy during a small interval $d t$ of time. After simplifying, one may get a relation of the type

$$
d E=n \Omega_{n} \tilde{r}_{A}^{n-1} \rho d \tilde{r}_{A}-n \Omega_{n} \tilde{r}_{A}^{n}(\rho+P) H d t
$$

iv) Using the relation $d \tilde{r}_{A}=-H \tilde{r}_{A}^{3}\left(\dot{H}-k / a^{2}\right) d t$ [10], we find that equations (12) and (13) result in a thermodynamical identity

$$
d E=T d S+W d V
$$

where $W=\frac{1}{2}(\rho-P)$. Here the quantity $W$ is nothing, but the work density defined in [17] through $W=-\frac{1}{2} T^{a b} h_{a b}$. Compared to the standard form of the first law of thermodynamics, $d E=T d S-P d V$, the work density $W$ replaces the pressure $P$ in our expression (14).

In the present work, we shall apply this procedure to the Friedmann equations in the Einstein gravity, Gauss-Bonnet gravity and Lovelock gravity at apparent horizon of FRW universe and show that the Friedmann equations in these gravities all can be rewritten as a universal thermodynamical identity (14). 


\section{THERMODYNAMIC BEHAVIOR OF FRIEDMANN EQUATION IN EINSTEIN GRAVITY}

The Einstein field equations read

$$
G_{\mu \nu}=8 \pi G T_{\mu \nu}
$$

where $G_{\mu \nu}$ is the Einstein tensor and $T_{\mu \nu}$ is the energy - momentum tensor of the matter fields. In the FRW universe, due to the symmetries of the FRW metric, the stress-energy tensor $T_{\mu \nu}$ must be diagonal, and by isotropy the spatial components must be equal. The simplest realization of such a stress-energy tensor is that of a perfect fluid described by a time dependent energy density $\rho(t)$ and pressure $P(t)$

$$
T_{\mu \nu}=(\rho+P) U_{\mu} U_{\nu}+P g_{\mu \nu}
$$

where $U^{\mu}$ is the four velocity of the fluid. With the conservation of stress-energy tensor $T_{; \nu}^{\mu \nu}=0$, one gets the continuity equation of the perfect fluid

$$
\dot{\rho}+n H(\rho+P)=0
$$

Solving Einstein's equations (15) in the background of metric (44) and assuming the energymomentum tensor (16) of perfect fluid, one gets the Friedmann equation

$$
H^{2}+\frac{k}{a^{2}}=\frac{16 \pi G}{n(n-1)} \rho
$$

where $H$ is the Hubble parameter with $H=\dot{a} / a$. Combining (17) and (18), one has $\dot{H}-\frac{k}{a^{2}}=$ $-\frac{8 \pi G}{n-1}(\rho+P)$. It can be seen from the radius $\tilde{r}_{A}=1 / \sqrt{H^{2}+k / a^{2}}$ of the apparent horizon that in the case of a flat universe, i.e., $k=0$, the radius $\tilde{r}_{A}$ of the apparent horizon has the same value as the radius $\tilde{r}_{H}$ of the Hubble horizon, which is defined as the inverse of the Hubble parameter, that is, $\tilde{r}_{H}=1 / H$. On the other hand, the cosmological event horizon defined by

$$
\tilde{r}_{E}=a(t) \int_{t}^{\infty} \frac{d t}{a(t)}
$$

exists only for an accelerated expanding universe. As a consequence, for a pure de Sitter universe with $k=0$, the apparent horizon, the Hubble horizon and the cosmological event horizon have the same constant value $1 / H$. Note that though the cosmological event horizon does not always exist for all FRW universes, the apparent horizon and the Hubble horizon always do exist. The apparent horizon has been argued to be a causal horizon for a dynamical space time and is associated with 
gravitational entropy and surface gravity [17, 18]. Thus for our purpose it would be useful to study the thermodynamical properties of Friedmann equations of FRW universe at the apparent horizon. (Note that in some cases, thermodynamics is not well-defined for Hubble horizon and event horizon [19]). In terms of the apparent horizon radius, the Friedmann equation (18) can be rewritten as

$$
\frac{1}{\tilde{r}_{A}^{2}}=\frac{16 \pi G}{n(n-1)} \rho .
$$

Then by taking differential of equation (20) and using the continuity equation (17), one gets the differential form of the Friedmann equation

$$
\frac{1}{\tilde{r}_{A}^{3}} d \tilde{r}_{A}=\frac{8 \pi G}{n-1}(\rho+P) H d t .
$$

Multiplying both hand sides of equation (21) by a factor $n \Omega_{n} \tilde{r}_{A}^{n}\left(1-\frac{\dot{\tilde{r}}_{A}}{2 H \tilde{r}_{A}}\right)$, one can rewrite this equation in the form

$$
\frac{\kappa}{2 \pi} d\left(\frac{n \Omega_{n} \tilde{r}_{A}^{n-1}}{4 G}\right)=-n \Omega_{n} \tilde{r}_{A}^{n}(\rho+P) H\left(1-\frac{\dot{\tilde{r}}_{A}}{2 H \tilde{r}_{A}}\right) d t .
$$

From the left hand side of equation (22), one immediately recognizes that the quantity $\frac{\kappa}{2 \pi}$ and the quantity $\frac{n \Omega_{n} \tilde{r}_{A}^{n-1}}{4 G}$ inside parentheses on the left hand side are nothing, but the temperature $T=\kappa / 2 \pi$ and entropy $S=A / 4 G\left(A=n \Omega_{n} \tilde{r}_{A}^{n-1}\right.$ being the area of the apparent horizon). Therefore the above equation can be rewritten as

$$
T d S=-n \Omega_{n} \tilde{r}_{A}^{n}(\rho+P) H\left(1-\frac{\dot{\tilde{r}}_{A}}{2 H \tilde{r}_{A}}\right) d t .
$$

Now we consider the Misner-Sharp energy (11) surrounded by the apparent horizon $\tilde{r}=\tilde{r}_{A}$ of the FRW universe, given by

$$
E=\frac{n(n-1) \Omega_{n}}{16 \pi G} \tilde{r}_{A}^{n-2}
$$

Using equation (20), one gets

$$
E=\Omega_{n} \tilde{r}_{A}^{n} \rho
$$

This is nothing, but the total energy $(\rho V)$ of matter inside the apparent horizon. It means that in Einstein gravity, the Misner-Sharp energy (11) surrounded by the apparent horizon is just the total energy of matter inside the apparent horizon. It no longer holds for Gauss-Bonnet gravity and Lovelock gravity. 
Taking differential of equation (25), we get

$$
d E=n \Omega_{n} \tilde{r}_{A}^{n-1} \rho d \tilde{r}_{A}+\Omega_{n} \tilde{r}_{A}^{n} d \rho
$$

Substituting $\dot{\rho}=-n H(\rho+P)$ into (26)

$$
d E=n \Omega_{n} \tilde{r}_{A}^{n-1} \rho d \tilde{r}_{A}-n \Omega_{n} \tilde{r}_{A}^{n}(\rho+P) H d t
$$

With the help of (27), equation (23) can be further rewritten to

$$
d E=T d S+\frac{1}{2} n \Omega_{n} \tilde{r}_{A}^{n-1}(\rho-P) d \tilde{r}_{A} .
$$

Note that the volume $V=\Omega_{n} \tilde{r}_{A}^{n}$ and let $W \equiv(\rho-P) / 2$. The above equation can be finally written to the form

$$
d E=T d S+W d V
$$

Comparing this with the standard form of the first law of thermodynamics, the negative pressure term is replaced by the term $W$. In fact, this is not strange, the result (29) is nothing, but the expression of the unified first law [17] of thermodynamics in the setup of FRW universe. In the second reference in [17], the author derived a similar formula for the trapping horizon of dynamical black hole. Here, it is important to note that the thermodynamic identity (29) is obtained by using the Friedmann equation of FRW universe together with the feature of apparent horizon.

In conclusion, by applying the Misner-Sharp energy relation, the Friedmann equation of FRW universe can be expressed as a thermodynamical identity $d E=T d S+W d V$ at the apparent horizon. On the other hand, from the relation (29) we may "derive" that the apparent horizon has an associated thermodynamics with temperature $T=\kappa / 2 \pi$ and entropy $S=A / 4 G$, where $\kappa$ is the surface gravity and $A$ is the area of apparent horizon.

\section{THERMODYNAMIC BEHAVIOR OF FRIEDMANN EQUATION IN GAUSS- BONNET GRAVITY}

In the previous section, we have studied the behavior of Friedmann equation at apparent horizon of FRW universe in Einstein gravity and identified its behavior as a thermodynamical system satisfying the unified first law of thermodynamics of the form $d E=T d S+W d V$. It has been found that the apparent horizon of FRW universe has an entropy proportional to its horizon area, very like the black hole horizon entropy obeying the so-called area formula [20]. However, it is well 
known that the area formula of black hole entropy no longer holds in higher derivative gravities. So it would be interesting to see whether, one can identify or not the Friedmann equation as a thermodynamical system near apparent horizon of FRW universe with entropy of expression with structure similar to the black hole horizon entropy in these gravities by the procedure developed in the previous section. In this section, we shall continue the previous procedure for a special form of higher derivative gravity, called Gauss-Bonnet gravity. This theory contains a special combination of curvature-squared term, added to the Einstein-Hilbert action. The Gauss-Bonnet term is given by

$$
R_{G B}=R^{2}-4 R_{\mu \nu} R^{\mu \nu}+R_{\mu \nu \gamma \delta} R^{\mu \nu \gamma \delta}
$$

The Gauss-Bonnet term naturally appears in the low energy effective action of heterotic string theory. The Gauss-Bonnet term is a topological term in four dimensions, and thus does not have any dynamical effect in these dimensions. The action of the Gauss-Bonnet gravity can be written by

$$
S=\frac{1}{16 \pi G} \int d^{n+1} x \sqrt{-g}\left(R+\alpha R_{G B}\right)+S_{m}
$$

where $R$ is the $(n+1)$-dimensional Ricci scalar, $S_{m}$ is the action of matter and $\alpha$ is a constant with the dimension $(\text { length })^{2}$. In the case of superstring theory in low energy limit $\alpha$ is related to the inverse string tension and is positive definite. The Gauss-Bonnet action is a natural extension of the Einstein theory in the sense that no derivatives higher than second order appear in the field equations. The equations of motion for the action (31) are given by

$$
G_{\mu \nu}+\alpha H_{\mu \nu}=8 \pi G T_{\mu \nu}
$$

where $G_{\mu \nu}=R_{\mu \nu}-\frac{1}{2} g_{\mu \nu} R$, and

$$
H_{\mu \nu}=2\left(R R_{\mu \nu}-2 R_{\mu \lambda} R_{\nu}^{\lambda}-2 R^{\gamma \delta} R_{\gamma \mu \delta \nu}+R_{\mu}^{\alpha \gamma \delta} R_{\alpha \nu \gamma \delta}\right)-\frac{1}{2} g_{\mu \nu} R_{G B} .
$$

In the vacuum Gauss-Bonnet gravity with/without a cosmological constant, static black hole solutions have been found and the associated thermodynamics has been discussed [21, 22]. In this theory, the static, spherically symmetric black hole has the form

$$
d s^{2}=-e^{\lambda(r)} d t^{2}+e^{\nu(r)} d r^{2}+r^{2} d \Omega_{n-1}^{2}
$$

with

$$
e^{\lambda(r)}=e^{-\nu(r)}=1+\frac{r^{2}}{2 \tilde{\alpha}}\left(1-\sqrt{1+\frac{64 \pi G \tilde{\alpha} M}{n(n-1) \Omega_{n} r^{n}}}\right)
$$


where $\tilde{\alpha}=(n-2)(n-3) \alpha$ and $M$ is the mass of black hole. In the limit $\alpha \rightarrow 0$, the above metric reduces to the Schwarzschild metric in Einstein gravity. The entropy of the black hole has the following form [22]

$$
S=\frac{A}{4 G}\left(1+\frac{n-1}{n-3} \frac{2 \tilde{\alpha}}{r_{+}^{2}}\right)
$$

where $A=n \Omega_{n} r_{+}^{n-1}$ is the horizon area and $r_{+}$is the horizon radius of the black hole. The authors of reference [10] have assumed that the entropy formula (36) also holds for the apparent horizon of FRW universe in the Gauss-bonnet gravity and the apparent horizon has the same expression for entropy but replacing the black hole horizon radius $r_{+}$by the apparent horizon radius $\tilde{r}_{A}$, i.e.,

$$
S=\frac{A}{4 G}\left(1+\frac{n-1}{n-3} \frac{2 \bar{\alpha}}{\tilde{r}_{A}^{2}}\right)
$$

with $A=n \Omega_{n} \tilde{r}_{A}^{n-1}$ being the area of the apparent horizon. They reproduced Friedmann equations by applying the relation $T d S=\delta Q$ to the apparent horizon with the assumption that the apparent horizon still has the horizon temperature $T=\frac{1}{2 \pi \tilde{r}_{A}}$. Now we show that the Friedmann equation of a FRW universe in the Gauss-Bonnet gravity also can be rewritten as the thermodynamic identity $d E=T d S+W d V$ at the apparent horizon.

The Friedmann equation for a FRW universe with perfect fluid as its source in the Gauss-Bonnet gravity is [23]

$$
\left(H^{2}+\frac{k}{a^{2}}\right)+\tilde{\alpha}\left(H^{2}+\frac{k}{a^{2}}\right)^{2}=\frac{16 \pi G}{n(n-1)} \rho .
$$

In terms of the apparent horizon radius, the Friedmann equation can be written as

$$
\frac{1}{\tilde{r}_{A}^{2}}+\tilde{\alpha} \frac{1}{\tilde{r}_{A}^{4}}=\frac{16 \pi G}{n(n-1)} \rho
$$

According to our procedure, one gets the differential form of the equation of motion by taking differential of equation (39)

$$
\frac{1}{\tilde{r}_{A}^{3}} d \tilde{r}_{A}+2 \tilde{\alpha} \frac{1}{\tilde{r}_{A}^{5}} d \tilde{r}_{A}=\frac{8 \pi G}{(n-1)}(\rho+P) H d t
$$

where the continuity equation, $\dot{\rho}=-n H(\rho+P)$, has been used. Now we multiply both hand sides of the above equation again by a factor $n \Omega_{n} \tilde{r}_{A}^{n}\left(1-\frac{\dot{\tilde{r}}_{A}}{2 H \tilde{r}_{A}}\right)$ and rewrite the above equation to the form

$$
\frac{\kappa}{2 \pi} d\left(\left(\frac{n \Omega_{n} \tilde{r}_{A}^{n-1}}{4 G}\right)\left(1+\frac{n-1}{n-3} \frac{2 \tilde{\alpha}}{\tilde{r}_{A}^{2}}\right)\right)=-n \Omega_{n} \tilde{r}_{A}^{n}(\tilde{\rho}+\tilde{P}) H\left(1-\frac{\dot{\tilde{r}}_{A}}{2 H \tilde{r}_{A}}\right) d t
$$


The first term in the left hand side of this equation (41) is in the form $T d S$. If we take

$$
\begin{aligned}
S & =\frac{n \Omega_{n} \tilde{r}_{A}^{n-1}}{4 G}\left(1+\frac{n-1}{n-3} \frac{2 \bar{\alpha}}{\tilde{r}_{A}^{2}}\right), \\
T & =\frac{\kappa}{2 \pi},
\end{aligned}
$$

then the equation (41) can be rewritten as

$$
T d S=-n \Omega_{n} \tilde{r}_{A}^{n}(\tilde{\rho}+\tilde{P}) H\left(1-\frac{\dot{\tilde{r}}_{A}}{2 H \tilde{r}_{A}}\right) d t .
$$

On the other hand, we note that the right hand side of the above equation is the same as the case in Einstein gravity (23). Thus one can immediately rewrite (43) as the thermodynamical identity

$$
d E=T d S+W d V
$$

Here we would like to remind the readers that in equation (44), the energy $E=\rho V$ is the total energy of matter (25) inside the apparent horizon, not the Misner and Sharp energy inside the apparent horizon. The reason is that the Misner and Sharp energy (24) cannot be written to the form (25) in the case of the Gauss-Bonnet gravity.

Thus, once again, we are able to express the Friedmann equation in the Gauss-Bonnet gravity as a thermodynamical identity (44), here the temperature and entropy associated with the apparent horizon are given by (42).

\section{THERMODYNAMIC BEHAVIOR OF FRIEDMANN EQUATIONS IN LOVELOCK GRAVITY}

In this section we extend the previous discussions to the more general Lovelock gravity. The Lovelock theory of gravity generalizes Einstein gravity when space time has a dimension greater than four. In this case the most general Lagrangian [24] that gives second order equations for the metric, is the sum over the dimensionally extended Euler densities

$$
L=\sum_{n=0}^{m} c_{n} L_{n},
$$

where $c_{n}$ is an arbitrary constant and $L_{n}$ is the Euler density of a 2n-dimensional manifold

$$
L_{n}=2^{-n} \delta_{c_{1} d \cdots c_{n} d_{n}}^{a_{1} b_{1} \cdots a_{n} b_{n}} R_{a_{1} b_{1}}^{c_{1} d_{1}} \cdots R_{a_{n} b_{n}}^{c_{n} d_{n}}
$$

where the generalized delta function $\delta_{c_{1} d \cdots c_{n} d_{n}}^{a_{1} b_{1} \cdots a_{n} b_{n}}$ is totally antisymmetric in both sets of indices and $R_{\alpha \beta}^{\gamma \delta}$ are the components of the curvature tensor. $L_{0}$ is set to one, therefore, the constant $c_{0}$ 
is just the cosmological constant. $L_{1}$ gives us the usual curvature scalar term. In order for the general relativity to be recovered in the low energy limit, the constant $c_{1}$ has to be positive. For simplicity, we can set $c_{1}=1 . L_{2}$ is just the Gauss-Bonnet term. Although the Lagrangian of the Lovelock gravity contains higher order derivatives curvature terms, there are no terms with more than second order derivatives of metric in equations of motion just as in Gauss-Bonnet gravity. Therefore, in this sense, the Lovelock gravity theory is not a higher derivative gravity theory. Lovelock field equations are much more complicated than Einstein's equations, but they can still be solved for some simple models as Friedmann universe and spherically symmetric black holes. The static spherically symmetric black hole solutions can be obtained in this theory in the sense that the metric function is determined by solving for a real root of a polynomial equation [21]. More recently, topological black hole solutions have been also found in the Lovelock gravity [25] (see also [26]). The horizon of these black holes can be hypersurface with a positive, zero or negative constant scalar curvature. In particular, it has been shown that the entropy of black hole horizon has a simple expression in terms of the horizon radius, while the expression for the metric function and causal structure of these black holes could be quite involved. For an $(n+1)$-dimensional static, spherically symmetric back hole with metric

$$
d s^{2}=-f(r) d t^{2}+f^{-1}(r) d r^{2}+r^{2} d \Omega_{n-1}^{2}
$$

the metric function is given by $f(r)=1-r^{2} F(r)$, where $F(r)$ is determined by solving for real roots of the following $m$ th-order polynomial equation

$$
\sum_{i=0}^{m} \hat{c}_{i} F^{i}(r)=\frac{16 \pi G M}{n(n-1) \Omega_{n} r^{n}} .
$$

Here, $M$ is a constant of integration, which is just the mass of the black hole, and the coefficients $\hat{c}_{i}$ are given by

$$
\hat{c}_{0}=\frac{c_{0}}{n(n-1)}, \quad \hat{c}_{1}=1, \quad \hat{c}_{i}=c_{i} \prod_{j=3}^{2 m}(n+1-j) \quad \text { for } \quad i>1
$$

The black hole entropy in terms of the horizon radius $r_{+}$can be expressed as [25]

$$
S=\frac{A}{4 G} \sum_{i=1}^{m} \frac{i(n-1)}{n-2 i+1} \hat{c}_{i} r_{+}^{2-2 i}
$$

where $A=n \Omega_{n} r_{+}^{n-1}$ is the horizon area of the black hole. The above expression of black hole entropy does not contain the cosmological constant term $c_{0}$ because the black hole entropy depends only upon the horizon geometry of the black hole. The entropy formula (50) of black hole also holds 
for the apparent horizon of FRW universe and the apparent horizon has the same expression for entropy in the Lovelock gravity but the black hole horizon radius $r_{+}$is replaced by the apparent radius $\tilde{r}_{A}[10]$. That is, the apparent horizon has the entropy

$$
S=\frac{A}{4 G} \sum_{i=1}^{m} \frac{i(n-1)}{n-2 i+1} \hat{c}_{i} \tilde{r}_{A}^{2-2 i}
$$

The Friedmann equation of a FRW universe in the Lovelock gravity is [10]

$$
\sum_{i=1}^{m} \hat{c}_{i}\left(H^{2}+\frac{k}{a^{2}}\right)^{i}=\frac{16 \pi G}{n(n-1)} \rho
$$

In terms of the apparent horizon radius $\tilde{r}_{A}^{2}=1 /\left(H^{2}+k / a^{2}\right)$, the Friedmann equation (52) can be rewritten as

$$
\sum_{i=1}^{m} \hat{c}_{i}\left(\tilde{r}_{A}\right)^{-2 i}=\frac{16 \pi G}{n(n-1)} \rho .
$$

One can get the differential form of the equation by taking differential of equation (53) and then using the continuity equation

$$
\sum_{i=1}^{m} i \hat{c}_{i}\left(\tilde{r}_{A}\right)^{-2 i-1} d \tilde{r}_{A}=\frac{8 \pi G}{(n-1)}(\rho+P) H d t
$$

We again multiply both hand sides of the above equation by $n \Omega_{n} \tilde{r}_{A}^{n}\left(1-\frac{\dot{\tilde{r}}_{A}}{2 H \tilde{r}_{A}}\right)$. And then arrange the left hand side, we have

$$
\frac{\kappa}{2 \pi} d\left(\frac{n \Omega_{n} \tilde{r}_{A}^{n-1}}{4 G} \sum_{i=1}^{m} \frac{i(n-1)}{n-2 i+1} \tilde{c}_{i} \tilde{r}_{A}^{2-2 i}\right)=-n \Omega_{n} \tilde{r}_{A}^{n}(\rho+P) H\left(1-\frac{\dot{\tilde{r}}_{A}}{2 H \tilde{r}_{A}}\right) d t .
$$

The left hand side of the above equation is of the form $T d S$. Thus the above equation can be rewritten as

$$
T d S=-n \Omega_{n} \tilde{r}_{A}^{n}(\rho+P) H\left(1-\frac{\dot{\tilde{r}}_{A}}{2 H \tilde{r}_{A}}\right) d t
$$

Once again, the right hand side of equation (56) has the same form as the case in Einstein gravity. Therefore we can finally rewrite the Freidmann equation into the universal form

$$
d E=T d S+W d V
$$

for the more general Lovelock gravity. Once again, here the energy $E$ is the total energy of matter inside the apparent horizon, not the Misner-Sharp energy, as the case of the Gauss-Bonnet gravity. 


\section{CONCLUSION AND DISCUSSION}

In this work we have shown that the differential form of Friedmann equation can be rewritten as a form, $d E=T d S+W d V$, at the apparent horizon of a FRW universe with any spatial curvature in arbitrary dimensions. Here $E$ the total energy $(\rho V)$ of matter inside the apparent horizon, $W=(\rho-P) / 2$ and $V$ is the volume inside the apparent horizon. Compared to the standard form of the first law of thermodynamics, the negative pressure term $-P$ is replaced by the work density $W$. Note that for pure de Sitter spacetime, $\rho=-P$, one then has a standard form $d E=T d S-P d V$. We have also shown that the Friedmann equations in the Gauss-Bonnet gravity and Lovelock gravity can also be expressed as the universal form. In particular, if associate a temperature $T=\kappa / 2 \pi$ to the apparent horizon, we can obtain an associated entropy $S=A / 4 G$ with the apparent horizon in the Einstein gravity, which has the same form as that of black hole entropy. In the Gauss-Bonnet gravity and Lovelock gravity, we have also obtained corresponding expressions of entropy, they keep the same forms as those of black hole entropy in each gravity. In other words, if we regard that the apparent horizon has a universal temperature $T=\kappa / 2 \pi$, we can pick up in our procedure the expression of entropy in different gravity theories. The resulting expressions of entropy have the same forms as obtained previously by using black hole thermodynamics. In addition, let us mention that in Einstein gravity, the total energy $E$ of matter inside the apparent horizon is just the Misner-Sharp energy, but they are not equal in Gauss-Bonnet gravity and Lovelock gravity.

Here more remarks are in order. First, it can be seen from (9) that if $\dot{\tilde{r}}_{A}<2 H \tilde{r}_{A}$, the apparent horizon has a negative surface gravity; if one further defines temperature $T=\kappa / 2 \pi$, the temperature is negative! This case is quite similar to the case of the cosmological event horizon in the Schwarzschild-de Sitter spacetime. In that case, one should define temperature $T=|\kappa| / 2 \pi$, and when the energy $E$ increases inside the apparent horizon, the apparent horizon radius $\tilde{r}_{A}$ decreases. The universal form should change to $-d E=T d S+W|d V|$ in this case. In addition, like the case of black hole spacetime, the temperature defined in this way only depends on the geometry, but not gravity theory under study. For the FRW universe, the apparent horizon has a universal expression $T=\kappa / 2 \pi$ with $\kappa$ given by (9).

Second, in [10], Cai and Kim have derived the Friedmann equations by applying the first law of thermodynamics, $T d S=-d E$, to the apparent horizon of a FRW universe with the assumption that the apparent horizon has temperature $T=1 / 2 \pi \tilde{r}_{A}$ and entropy $S=A / 4 G$. One might worry that the result in [10] is not consistent with the one in the present paper. This is not the case, in 
fact, they are consistent with each other. To be not confused, first, we would like to stress here that the notation $d E$ in [10] is quite different from the same one used in the present manuscript. In [10], $-d E$ is actually just the heat flux $\delta Q$ in [9] crossing the apparent horizon within an infinitesimal internal of time $d t$. The quantity is given by

$$
\delta Q=-d E=n \Omega_{n} \tilde{r}_{A}^{n}(\rho+P) H d t .
$$

In this calculation, the apparent horizon radius has been assumed to be fixed. In this manuscript we have used the matter energy $E$ given in (25) inside the apparent horizon. We have assumed that $d \tilde{r}_{A}$ be the infinitesimal change in the radius of the apparent horizon in a small interval of time $d t$ which causes a small change $d V$ in volume of the apparent horizon. Since the matter energy $E$ is directly related with the radius of the apparent horizon, therefore, the change of apparent horizon radius will change the energy $d E$ inside the apparent horizon. By this procedure, we worked out the change of energy $d E$ inside the apparent horizon is

$$
d E=n \Omega_{n} \tilde{r}_{A}^{n-1} \rho d \tilde{r}_{A}-n \Omega_{n} \tilde{r}_{A}^{n}(\rho+P) H d t
$$

Therefore in our definition, a new term $n \Omega_{n} \tilde{r}_{A}^{n-1} \rho d \tilde{r}_{A}$ appears. Since the apparent horizon radius is assumed to be fixed in calculating (58) in [10] (see also [12, 13, 14]), from (9) the temperature therefore has the form $T=1 / 2 \pi \tilde{r}_{A}$ in that case. Furthermore, a natural consequence is that the term of volume change is absent in [10].

Third, one interesting question may arise; whether one can always express the Friedmann equations to the thermodynamic identity $T d S=d E+W d V$ at apparent horizon in any gravity theory? Since in Einstein, Gauss-Bonnet and more general Lovelock gravity theories, the fact that the Friedmann equations can be rewritten to the universal form, $d E=T d S+W d V$, might be related to the observation that in these gravities, the equations of field can be derived from a holographic surface term [27]. If it is possible, is it always possible to pick up the expressions for entropy from the identity $d E=T d S+W d V$, which agree with previously known results? For example, in cases of $f(R)$ and scalar tensor gravities, can one apply this approach to identify field equations as the universal thermodynamic form at apparent horizon and pick up the expressions for entropies in these gravities? Partial results of these issues are obtained recently [28].

Finally, one more question is whether this is a procedure applicable for all types of horizons of space-times? These issue are certainly associated with the holographic properties of gravity. It would be of great interest to examine further the consequences of these observations to the holographic principle. 


\section{Acknowledgments}

The authors thank Y.G. Gong and T. Padmanabhan for helpful correspondences. The work was supported in part by a grant from Chinese Academy of Sciences, by NSFC under grants No. 10325525 and No. 90403029.

[1] S. W. Hawking, Commun. Math. Phys. 43, 199 (1975).

[2] J. D. Bekenstein, Phys. Rev. D7, 2333 (1973).

[3] J. M. Bardeen, B. Carter and S. W. Hawking, Commun. Math. Phys. 31, 161 (1973).

[4] J. Bekenstein, "Do we understand black hole entropy"? arXiv: gr-qc/9409015; U.H. Gerlach, Phys. Rev. D15, 1479 (1976); G. t’Hooft, Nucl. Phys. B256, 727 (1985); J. York, Phys. Rev. D15, 2929 (1985); W.H. Zurek and K.S. Thorne, Phys. Rev. Lett. 54, 2171 (1985); L. Bombelli et al., Phys. Rev. D34,373(1986); R. D. Sorkin, arXiv: gr-qc/9705006.

[5] A. Paranjape, S. Sarkar and T. Padmanabhan, arXiv:hep-th/0607240.

[6] T. Padmanabhan, Class. Quant. Grav. 19, 5387 (2002)[arXiv: gr-qc/0204019]; Phys. Rept. 406, 49 (2005) [arXiv: gr-qc/0311036]; T. Padmanabhan, arXiv:gr-qc/0606061.

[7] G. W. Gibbons and S. W. Hawking, Phys. Rev. D15, 2738 (1977).

[8] R. G. Cai, Nucl. Phys. B628, 375(2002) [arXiv: hep-th/0112253]; R. G. Cai, Phys. Lett. B525, 331(2002) [arXiv:hep-th/0111093].

[9] T. Jacobson, Phys. Rev. Lett. 75, 1260(1995)[arXiv: hep-th/0212327].

[10] R. G. Cai and S. P. Kim, JHEP 0502, 050 (2005)[arXiv:hep-th/0501055]

[11] M. Akbar and R. G. Cai, Phys. Lett. B635, 7 (2006). [arXiv:hep-th/0602156]

[12] A. V. Frolov and L. Kofman, JCAP 0305, 009 (2003).

[13] U. K. Danielsson, Phys. Rev. D71, 023516(2005)[arXiv: hep-th/0411172].

[14] R. Bousso, Phys. Rev. D71, 064024(2005)[arXiv: hep-th/0412197].

[15] G. Calcagni, JHEP 0509, 060 (2005) [arXiv:hep-th/0507125].

[16] C.W. Misner and D. H. Sharp, Phys. Rev. 136, B571 (1964).

[17] S. A. Hayward, S. Mukohyana, and M. C. Ashworth, Phys. Lett. A256, 347(1999); S. A. Hayward, Class. Quant. Grav. 15, 3147 (1998) [arXiv:gr-qc/9710089].

[18] D. Bak and S. J. Rey, Class. Quant. Grav. 17, L83 (2000).

[19] B. Wang, Y. Gong and E. Abdalla, arXiv:gr-qc/0511051.

[20] R. M. Wald, Phys. Rev. D48, 3427(1993).

[21] D. G. Boulware and S. Deser, Phys. Rev. Lett. 55, 2656 (1985); J. T. Wheeler, Nucl. Phys. B 268, 737 (1986); Nucl. Phys. B 273, 732 (1986); R. C. Myers and J. Z. Simon, Phys. Rev. D 38, 2434 (1988).

[22] R. G. Cai, Phys. Rev. D65, 084014 (2002); R. G. Cai and Q. Guo, Phys. Rev. D69, 104025 (2004). 
[23] R. G. Cai and Y. S. Myang, Phys. Lett. B559, 60(2003).

[24] D. Lovelock, J. Math. Phys. 12, 498 (1971).

[25] R. G. Cai, Phys. Lett. B582, 237(2004)[arXiv: hep-th/0311240].

[26] M. Banados, C. Teitelboim and J. Zanelli, Phys. Rev. D 49, 975 (1994) [arXiv:gr-qc/9307033]; R. G. Cai and K. S. Soh, Phys. Rev. D 59, 044013 (1999) [arXiv:gr-qc/9808067]; J. Crisostomo, R. Troncoso and J. Zanelli, Phys. Rev. D 62, 084013 (2000) [arXiv:hep-th/0003271]; R. Aros, R. Troncoso and J. Zanelli, Phys. Rev. D 63, 084015 (2001) [arXiv:hep-th/0011097].

[27] A. Mukhopadhyay and T. Padmanabhan, arXiv:hep-th/0608120.

[28] M. Akbar and R.G. Cai, gr-qc/0612089; R.G. Cai and L.M Cao, gr-qc/0611071; R.G. Cai and L.M. Cao, hep-th/0612144. 